\title{
Steroid Myopathy
}

National Cancer Institute

\section{Source}

National Cancer Institute. Steroid Myopathy. NCI Thesaurus. Code C88134.

A non-neoplastic disorder affecting the skeletal muscles. It is caused by damage to muscle fibers either by excessive intake of corticosteroids (steroid treatment) or high levels of endogenous corticosteroids due to hormonal abnormalities. Patients usually present with weakness mainly in the proximal muscles of the upper and lower extremities and the neck flexors. 